Case Report

\title{
A Case of Subacute Cutaneous Lupus Erythematosus in a Patient with Mixed Connective Tissue Disease: Successful Treatment with Plasmapheresis and Rituximab
}

\author{
M. Fantò, ${ }^{1}$ S. Salemi, ${ }^{1}$ F. Socciarelli, ${ }^{2}$ A. Bartolazzi, ${ }^{2}$ G. A. Natale, ${ }^{3}$ I. Casorelli, ${ }^{3}$ A. Pavan, ${ }^{3}$ \\ S. Vaglio, ${ }^{3}$ R. Di Rosa, ${ }^{1}$ and R. D'Amelio ${ }^{1}$ \\ ${ }^{1}$ Department of Allergy, Clinical Immunology and Rheumatology, S. Andrea Hospital, Sapienza University of Rome, Italy \\ ${ }^{2}$ Department of Pathology, S. Andrea Hospital, Sapienza University of Rome, Italy \\ ${ }^{3}$ Department of Immunohematology and Transfusion Unit, S. Andrea Hospital, Sapienza University of Rome, Italy
}

Correspondence should be addressed to M. Fantò; martafanto@gmail.com

Received 4 June 2013; Accepted 26 June 2013

Academic Editors: L.-P. Erwig, M. A. Hunt, and M. Soy

Copyright @ 2013 M. Fantò et al. This is an open access article distributed under the Creative Commons Attribution License, which permits unrestricted use, distribution, and reproduction in any medium, provided the original work is properly cited.

\begin{abstract}
A 30-year-old woman affected by Mixed Connective Tissue Disease with scleroderma spectrum developed a facial eruption, a clinical and histological characteristic of subacute cutaneous lupus erythematosus (SCLE). Speckled anti-nuclear antibodies, high-titer anti-ribonucleoproteinl, anti-Sm, anti-Cardiolipin (aCL) IgG/IgM, and anti-Ro/SSA antibodies were positive. SCLE was resistant to Azathioprine, Hydroxychloroquine, and Methotrexate while Mycophenolate Mofetil was suspended due to side effects. Subsequently, the patient was treated with three cycles of therapeutic plasma exchange (TPE) followed, one month after the last TPE, by the anti-CD20 antibody Rituximab (RTX) $\left(375 \mathrm{mg} / \mathrm{m}^{2}\right.$ weekly for 4 weeks). Eight and 16 months later the patient received other two TPE and RTX cycles, respectively. This therapeutic approach has allowed to obtain a complete skin healing persistent even after 8-month follow-up. Moreover, mitigation of Raynaud's phenomenon, resolution of alopecia, and a decline of aCL IgG/IgM and anti-Ro/SSA antibodies were observed.
\end{abstract}

\section{Introduction}

Mixed Connective Tissue Disease (MCTD) is currently defined as an overlapping syndrome with clinical features of Systemic Sclerosis (SSc), Systemic Lupus Erythematosus (SLE), Rheumatoid arthritis (RA), and Polymyositis/Dermatomyositis (PM/DM); the presence of high-titer antiribonucleoproteinl (U1RNP) or speckled anti-nuclear antibodies (ANA) at titer $\geq 1: 2,000$ is necessary for the diagnosis. The disease affects mainly women in the third decade of life (from 80 to $90 \%$ ) but it has been also reported in children and in over-80-year-old people [1].

The most frequent clinical manifestations are Raynaud's phenomenon (RP), swollen hands, sclerodactyly, arthritis, myalgias, and oesophageal dysmotility, and also alopecia, malar rash, lymphadenopathy, or kidney damage can be present. Rarely, subacute cutaneous lupus erythematosus (SCLE), characterized by annular or papulosquamous lesions, photosensitivity, and presence of anti-Ro/SSA and anti-La/SSB antibodies, has been described in MCTD patients $[2,3]$. MCTD therapy should be identified for each patient depending on the affected organ, but generally there is a good response to steroids, different types of vasodilators, and immunosuppressive agents such as Hydroxychloroquine (HCQ), Azathioprine (AZA), Methotrexate (MTX), or Cyclophosphamide (CYC) [1].

\section{Case Presentation}

A case of a thirty-year-old woman affected by MCTD with scleroderma spectrum and epilepsy since she was fifteen is here reported. At the beginning she presented fever up to 


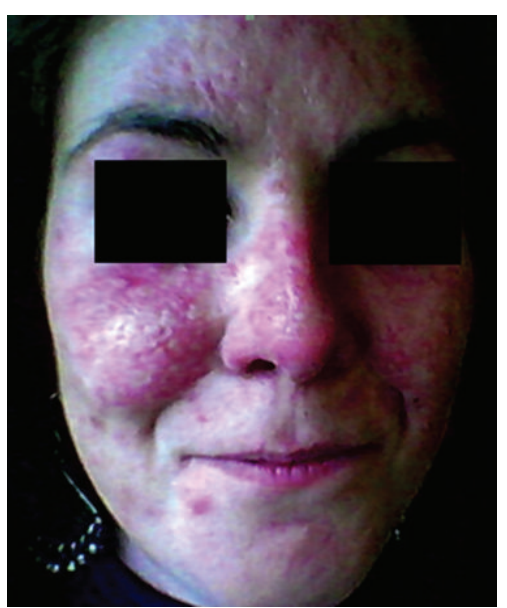

(a)

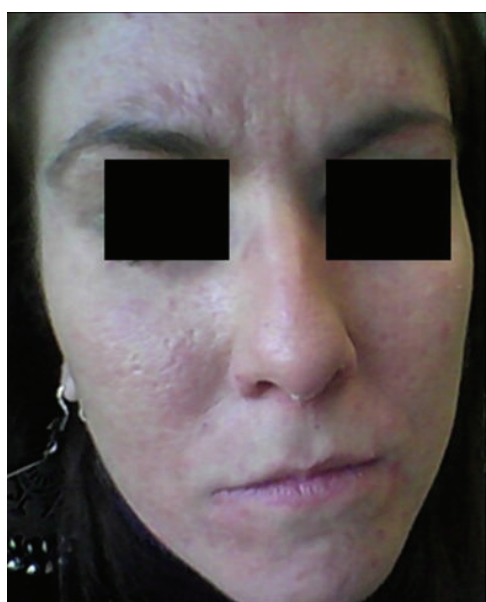

(b)

FIGURE 1: (a) SCLE: cutaneous eruption which infiltrates forehead, cheeks, and chin. (b) Disappearance of facial SCLE after the third cycle of TPE plus RTX.

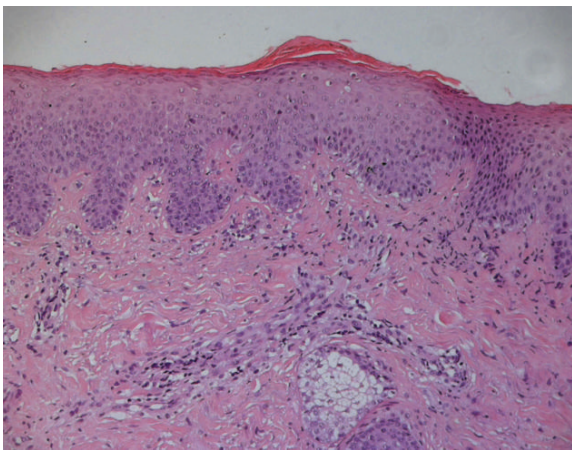

(a)

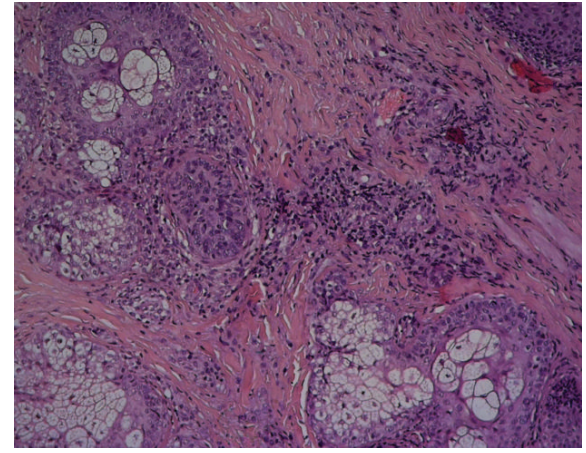

(b)

Figure 2: (a) The epidermal layer is characterized by a mild degree of papillomatosis, acanthosis, and focal mixed orthokeratotic and parakeratotic hyperkeratoses. The underlying papillary and reticular dermis shows a marked fibrotic change associated with a chronic mononuclear perivascular inflammatory infiltrate (H\&E, 100x magnification). (b) A moderate mononuclear chronic infiltrate is present around adnexal structures (H\&E, 100x magnification).

$40^{\circ} \mathrm{C}$, arthalgias mainly at knees, wrists, and shoulders, and increased levels of erythrocyte sedimentation rate (ESR) and C-reactive protein (CRP). She also had speckled type of ANA up to $1: 2,560$, anti-U1RNP, anti-Sm, anti-Cardiolipin $(\mathrm{aCl})$ IgG and IgM positivity, hypergammaglobulinemia, myositis, lymphopenia, RP, cutaneous calcinosis, and scleroderma. She started treatment with Cyclosporine A (CYA), corticosteroids (CCS), and nifedipine in 1998. The following year myositis worsened with an increase of Creatinphosphokinase (CPK) up to 8,000; thus she received pulse steroid therapy, $800 \mathrm{mg} /$ die methylprednisolone, monthly for six months; four years later, in 2001, because of exacerbation of arthralgias she started HCQ, with satisfying improvement. In August 2003 a grade $\mathrm{C}$ esophagitis and a diffuse bilateral interstitial lung disease with severe decrease of carbon monoxide diffusing capacity (DLCO) were detected. Irregular urticarial lesions in her arms and chest and purpura in her legs and alopecia also arose. Thus she started AZA, with lung and cutaneous improvement. In 2007 CYA was suspended after a blood pressure increase. Subsequently, a facial eruption appeared in correspondence of forehead, cheeks, and chin (Figure 1(a)). Histopathological examination of a skin biopsy revealed "a skin characterized by modest papillomatosis, acanthosis, and focal hyperkeratosis of the epidermis. The superficial and deep dermis showed marked sclerosis associated with lymphomononuclear perivascular and periadnexal cellular infiltrate" (Figures 2(a)-2(b)).

Direct immunofluorescence on frozen skin biopsy ("lupus band test") demonstrated "a dust-like IgG particles staining pattern consisting of fine granular Ig deposition scattered through the epidermis." This picture has been reported to be specific for SCLE [4]. Taking into account the clinical and histological features and anti/Ro antibodies positivity, a diagnosis of subacute cutaneous lupus erythematosus (SCLE) associated to dermal sclerosis was made. In 2008 MTX and the subsequent year Mycophenolate Mofetil (MMF) were able to induce a slight skin improvement but they were stopped due to inefficacy and excessive weight 
loss, respectively. Therefore, in December 2010, therapeutic plasma exchange (TPE) was performed, every other day for a total of five exchanges, using albumin to replace the plasma removed. The same cycle was repeated in February and in March 2011 followed, one month after, by Rituximab (RTX) ( $375 \mathrm{mg} / \mathrm{m}^{2}$ weekly for 4 weeks). Eight and 16 months later the patient received other two TPE procedures followed by RTX (375 mg $/ \mathrm{m}^{2}$ weekly for 4 weeks), respectively. After the first 3 TPE cycles there was a slight improvement of SCLE and the addition of RTX has allowed for obtaining a further clearing of facial eruption, while 8 weeks after the last RTX infusion a complete skin healing was reached and is still persistent after 8-month follow-up (Figure 1(b)). Mitigation of RP and resolution of alopecia were also observed. During this period no flares-up were observed and the patient assumed only HCQ and a low-dose of CCS $(5 \mathrm{mg} /$ die). Interestingly, aCl-IgG/IgM and anti-Ro/SSA antibodies disappeared after the first TPE and RTX treatment, whereas they showed a slight increase before the third cycle at the end of which they became definitively negative for the next 8 months.

\section{Discussion}

Successful off-label use of RTX in SLE manifestations as cytopenia, diffuse erythematosus lesions, and alopecia or as rescue therapy in life-threatening complications of several autoimmune diseases has been sometimes reported $[5,6]$; in addition, the anti-CD20 therapy has even been employed in dermatologic field including blistering diseases, graft versus host disease, and DM/PM but, to our knowledge, only one case of refractory SCLE treated with RTX has been described [7]. Recently, the role of B cells in the pathogenesis of SSc has been underlined, and the RTX efficacy to improve skin fibrosis and pulmonary function has been reported $[8,9]$.

Regarding MCTD, a successful treatment with RTX (in combination with CCS, CYC, and iloprost) has been reported in a case of severe, refractory RP [10], while on the contrary, Dunkley et al. reported a case of MCTD with scleroderma spectrum in which RTX was not able to control RP [11]. Moreover two MCTD cases in which TPE was able to treat visceral RP with multiple organ damage (treated with combination with CYA and CCS) [12] and acute renal failure (in combination with CYC and captopril) [13] have been described.

Plasmapheresis, in association with RTX, has been used in only one case of MCTD patient, in whom were observed $\mathrm{RP}$ resolution, ANA, anti-centromere (CENP-B) antibodies, and decrease of serum IgG-IgM [14].

A case of MCTD patient with scleroderma spectrum, in whom therapy-resistant facial SCLE was completely resolved after combination of TPE and RTX $\left(375 \mathrm{mg} / \mathrm{m}^{2}\right.$ weekly for 4 weeks), is here reported. Moreover, we observed RP improvement and alopecia, $\mathrm{aCl}$, and anti-Ro/SSA serum antibodies disappearance. Anti-Ro/SSA antibodies are closely associated with SCLE, and the resolution of clinical features accompanied by the disappearance of these antibodies from serum strongly suggests a primary role of anti-Ro/SSA autoantibodies in the pathogenesis of SCLE.

Before starting this new approach, in order to exclude a iatrogenic cause in SCLE induction, carbamazepine and nifedipine were replaced with similar noninducing drugs, but no clinical effects on SCLE were observed [15].

In conclusion, association of TPE and RTX should be considered as a valid and safe therapeutic tool for controlling SCLE in therapy-resistant MCTD. Moreover, despite the relative short follow-up, the intriguing observation of the beneficial effects that this treatment could exert also on cutaneous sclerosis occurring in MCTD patients makes this therapeutic approach very promising.

\section{References}

[1] O.-D. Ortega-Hernandez and Y. Shoenfeld, "Mixed connective tissue disease: an overview of clinical manifestations, diagnosis and treatment," Best Practice and Research, vol. 26, no. 1, pp. 6172, 2012.

[2] A. Parodi, M. Caproni, C. Cardinali et al., "Clinical, histological and immunopathological features of 58 patients with subacute cutaneous lupus erythematosus. A review by the Italian Group of Immunodermatology," Dermatology, vol. 200, no. 1, pp. 6-10, 2000.

[3] D. Lipsker, M.-P. Di Cesare, B. Cribier, E. Grosshans, and E. Heid, "The significance of the "dust-like particles" pattern of immunofluorescence. A study of 66 cases," British Journal of Dermatology, vol. 138, no. 6, pp. 1039-1042, 1998.

[4] K. M. David-Bajar, S. D. Bennion, J. D. DeSpain, L. E. Golitz, and L. A. Lee, "Clinical, histologic, and immunofluorescent distinctions between subacute cutaneous lupus erythematosus and discoid lupus erythematosus," Journal of Investigative Dermatology, vol. 99, no. 3, pp. 251-257, 1992.

[5] M. Ramos-Casals, M. J. Soto, M. J. Cuadrado, and M. A. Khamashta, "Rituximab in systemic lupus erythematosus: a systematic review of off-label use in 188 cases," Lupus, vol. 18, no. 9, pp. 767-776, 2009.

[6] Y. Braun-Moscovici, Y. Butbul-Aviel, L. Guralnik et al., "Rituximab: rescue therapy in life-threatening complications or refractory autoimmune diseases: a single center experience," Rheumatology International, vol. 33, no. 6, pp. 1495-1504, 2013.

[7] V. Kieu, T. O’Brien, L.-M. Yap et al., "Refractory subacute cutaneous lupus erythematosus successfully treated with rituximab," Australasian Journal of Dermatology, vol. 50, no. 3, pp. 202-206, 2009.

[8] V. Smith, Y. Piette, J. T. van Praet et al., "Two-year results of an open pilot study of a 2 -treatment course with rituximab in patients with early systemic sclerosis with diffuse skin involvement," The Journal of Rheumatology, vol. 40, pp. 52-57, 2013.

[9] D. Daoussis, S. N. Liossis, A. C. Tsamandas et al., "Effect of longterm treatment with rituximab on pulmonary function and skin fibrosis in patients with diffuse systemic sclerosis," Clinical and Experimental Rheumatology, vol. 30, no. 2, supplement 71, pp. S17-S22, 2012.

[10] M. Haroon, D. O'Gradaigh, and D. Foley-Nolan, "A case of Raynaud's phenomenon in mixed connective tissue disease responding to rituximab therapy," Rheumatology, vol. 46, no. 4, pp. 718-719, 2007. 
[11] L. Dunkley, M. Green, and A. Gough, "Comment on: a case of Raynaud's phenomenon in mixed connective tissue disease responding to Rituximab therapy-response," Rheumatology, vol. 46, no. 10, pp. 1628-1629, 2007.

[12] M. Seguchi, Y. Soejima, A. Tateishi et al., "Mixed connective tissue disease with multiple organ damage: successful treatment with plasmapheresis," Internal Medicine, vol. 39, no. 12, pp. 1119$1122,2000$.

[13] R. M. Crapper, J. P. Dowling, I. R. Mackay, and J. A. Whitworth, "Acute scleroderma in stable mixed connective tissue disease: treatment by plasmapheresis," Australian and New Zealand Journal of Medicine, vol. 17, no. 3, pp. 327-329, 1987.

[14] J. Rech, S. Kallert, A. J. Hueber, C. Requadt, J. R. Kalden, and H. Schulze-Koops, "Combination of immunoadsorption and CD20 antibody therapy in a patient with mixed connective tissue disease," Rheumatology, vol. 45, no. 4, pp. 490-491, 2006.

[15] G. Lowe, C. L. Henderson, R. H. Grau, C. B. Hansen, and R. D. Sontheimer, "A systematic review of drug-induced subacute cutaneous lupus erythematosus," British Journal of Dermatology, vol. 164, no. 3, pp. 465-472, 2011. 


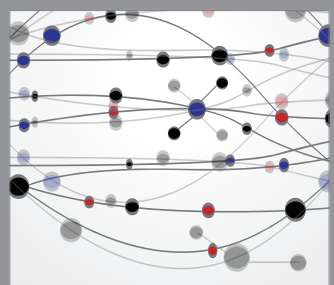

The Scientific World Journal
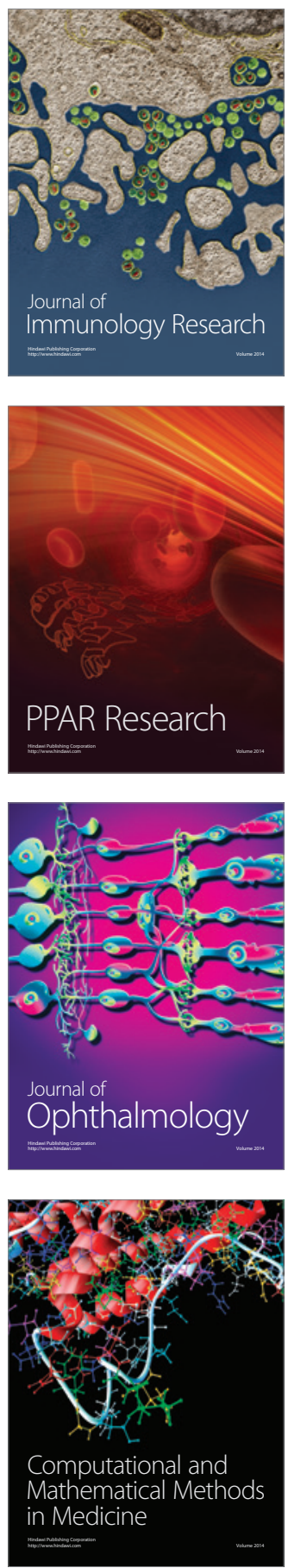

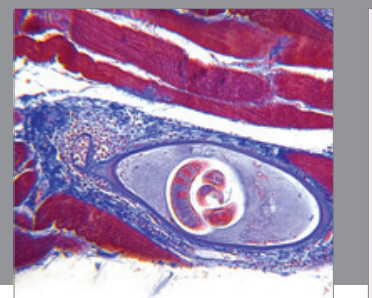

Gastroenterology

Research and Practice
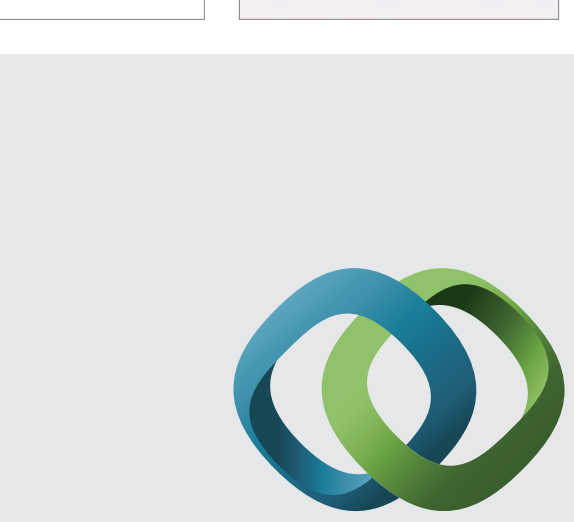

\section{Hindawi}

Submit your manuscripts at

http://www.hindawi.com
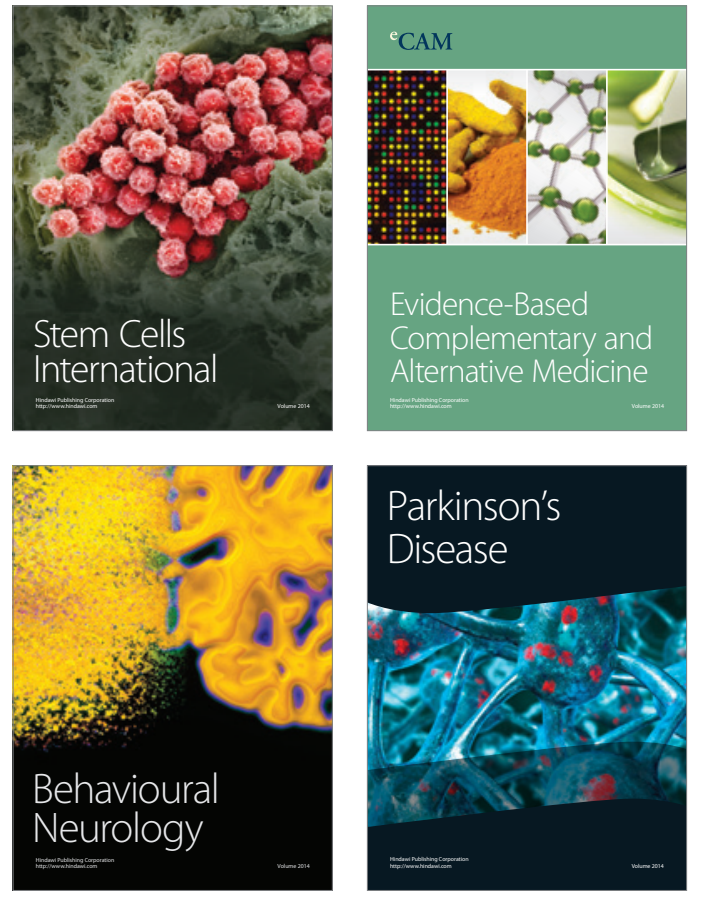
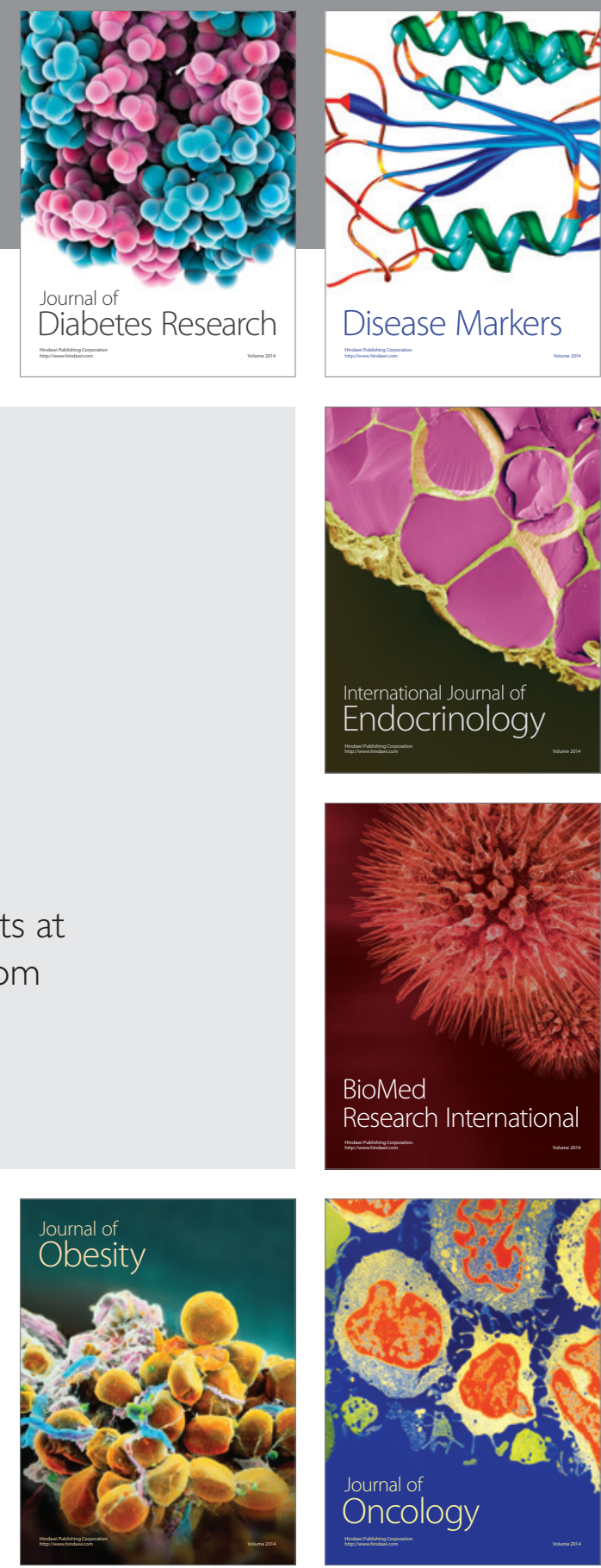

Disease Markers
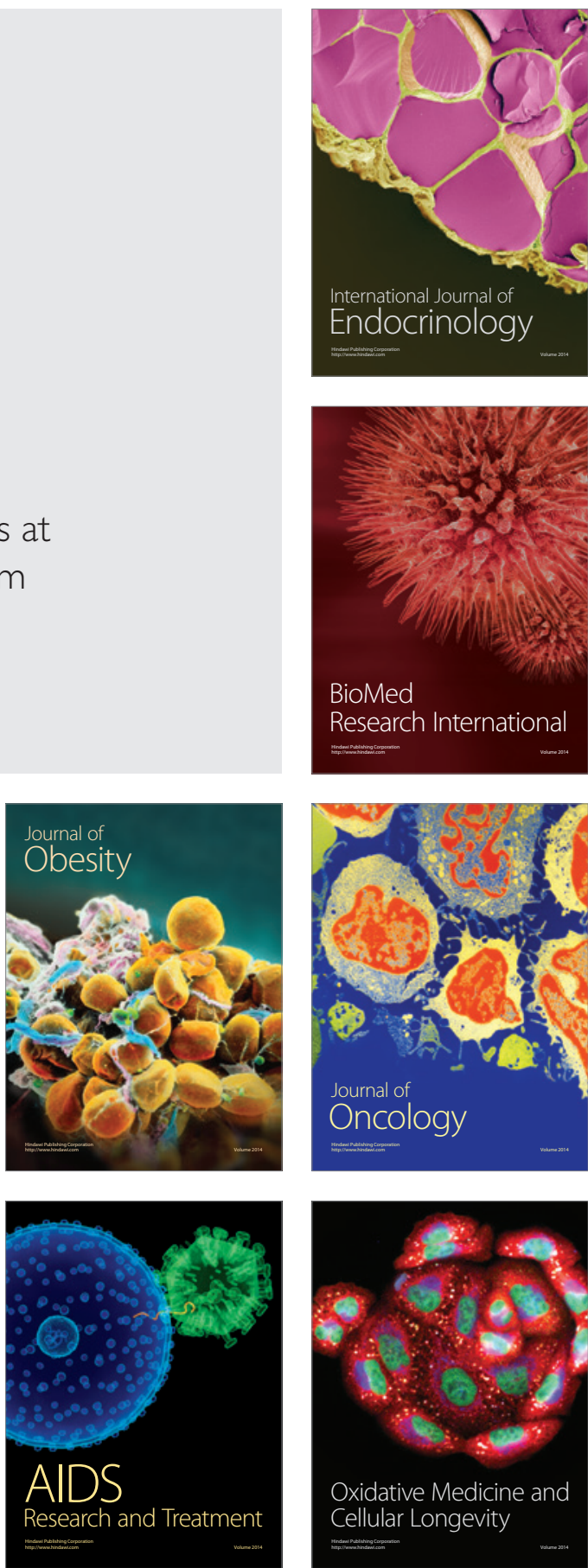